\title{
Spatially-temporal dynamics of a passively Q-switched Raman-active solid-state oscillator
}

\author{
V.L.Kalashnikov*,a,1, A.M.Malyarevich ${ }^{\mathrm{b}}$, K.V.Yumashev ${ }^{\mathrm{b}}$ \\ ${ }^{a}$ Institut für Photonik, TU Wien, Gusshausstr. 27/387, A-1040 Vienna, Austria \\ ${ }^{b}$ Institute for Optical Materials and Technologies, 65 Nezavisimosti Ave., Bldg. 17, \\ 220013 Minsk, Belarus
}

\begin{abstract}
The spatially-temporal model of an all-solid-state passively Q-switched oscillator with an active medium providing the stimulated Raman scattering is presented. The model does not presume a Gaussian shape of the cylindrically symmetric modes at both fundamental and Stokes wavelengths. It is found, that the highly-nontrivial spatially-temporal dynamics can be regularized by the optimal choice of the oscillator parameters, viz. initial transmission of a saturable absorber, curvature of a spherical mirror, and output mirror transmission at the fundamental and Stokes wavelengths. As a result, the pulse can be substantially temporally squeezed and spatially broadened at both fundamental and Stokes wavelengths.
\end{abstract}

Key words: Q-switching; Solid-state lasers; stimulated Raman scattering; spatially-temporal dynamics

PACS: 42.60.Gd;42.55.Ye;42.65.Sf

\section{Introduction}

Solid-state Q-switched oscillators allowing nano- and sub-nanosecond pulsing find applications in a lot of areas including medicine, spectroscopy, environment monitoring, etc. Passive Q-switching based on the use of both

\footnotetext{
${ }^{*}$ Corresponding author

Email address: kalashnikov@tuwien.ac.at (V.L.Kalashnikov)

URL: http://info.tuwien.ac.at/kalashnikov (V.L.Kalashnikov)

${ }^{1}$ Author acknowledges the support from the Austrian Fonds zur Forderung der wissenschaftlichen Forschung (project P20293).
} 
semiconductor [1, 2] and crystalline [3] saturable absorbers is of particular interest due to compactness, simplicity, high damage threshold, and diodepumping ability of an oscillator.

Among the active media allowing diode-pumped Q-switching, $\mathrm{KY}\left(\mathrm{WO}_{4}\right)_{2}$ $(\mathrm{KYW})$ and $\mathrm{KGd}\left(\mathrm{WO}_{4}\right)_{2}(\mathrm{KGW})$ crystals doped by $\mathrm{Yb}^{3+}$ and $\mathrm{Nd}^{3+}$ ions are known as the materials providing an efficient stimulated Raman scattering (SRS) [4, 5, 6, 7, 8]. As a result, there is possible a high-efficient selffrequency shift and a simultaneous two-wavelengths pulsing (e.g., at 1.35 and $1.54 \mu \mathrm{m}$ for a Nd:KGW active medium) directly from an oscillator.

Theoretical studies of a Q-switched oscillator with the intra-cavity SRS have been based on the well-established rate-equations approach [9, 10, 11]. The oscillator parameters providing a pulse width minimization [10] and an output energy maximization [11] at both fundamental and Stokes wavelengths have been defined. Simultaneously, it has been found that the spatial structure of a laser field is strongly affected by the SRS [9]. This means that the mode transformation has to be taken into account along with the temporal evolution of fields and populations inside an active medium and a saturable absorber. Such a model has been developed to a Gaussian mode approximation for both fundamental and Stokes fields [12]. This has allowed defining the optimal values of the Raman gain and the ratio of pump and laser beams, which provide a single pulse operation of oscillator with the most efficient SRS.

Nevertheless, a transient character of Q-switching can prevent from the CW mode formation and disturb substantially the spatial structure of a laser field. This requires to take into account the spatial dynamics on a par with the temporal one.

In this work we present the analysis of spatially-temporal dynamics of a passively Q-switched Raman-active oscillator. The analysis is based on a rate-equations approach but without imposing a limitation on the shape of cylindrically symmetric transverse distribution of a field. The results demonstrate that there exist both spatial extra-broadening and squeezing as well as transition between non-Gaussian and Gaussian spatial profiles in dependence on the oscillator parameters (viz. output mirror transmission at the fundamental and Stokes wavelengths, initial transmission of a saturable absorber and curvature of a spherical mirror). The SRS contribution controlled by the optimized sets of oscillator parameters allows the regularization of the spatially-temporal structure of a field and the substantial pulse shortening. 


\section{Numerical procedure}

The oscillator under consideration consists of the flat (output) and spherical mirrors. The active medium $\left(\mathrm{Nd}^{3+}: \mathrm{KGW}\right)$ is placed at the resonator center, and the saturable absorber $\left(\mathrm{V}^{3+}: \mathrm{YAG}\right)$ is placed on the output mirror.

The model is based on a direct generalization of that presented in [10, 12]. It is supposed that the time-dependent ( $t$ is the time, which is periodical with the cavity period $T_{c a v}: t \in\left[0, T_{c a v}\right]$ ) fields $a_{f, s}$ (indexes $f$ and $s$ correspond to the fundamental and Stokes fields, respectively) have the radially-symmetric transverse spatial distributions $(r \in] 0, R]$ is the radial coordinate).

Inside an active medium, the dynamics is modeled on basis of the splitstep Hankel's method. That is the active medium volume is considered to be divided into 10 transverse slices with the thickness $\Delta z$ ( $z$ is the longitudinal coordinate), in which the dynamics within the time-domain and the domain of spatial frequencies $(\nu \equiv c / 2 \pi R, c$ is the velocity of light, $R=0.5 \div 1.5$ cm) [13] is evolved step-by-step:

$$
\begin{gathered}
\dot{a}_{f}(z, r, t)=\frac{\Delta z}{2 T_{c a v}} a_{f}(z, r, t)\left[g(r, t)-g_{s}\left|a_{s}(z, r, t)\right|^{2}\right] \\
\dot{a}_{s}(z, r, t)=\frac{\Delta z}{2 T_{c a v}} a_{s}(z, r, t) g_{s}\left|a_{f}(z, r, t)\right|^{2} \\
\dot{g}(r, t)=-\frac{\sigma_{g} \lambda_{f}}{h c} g(r, t)\left|a_{f}(z, r, t)\right|^{2} \\
\otimes \tilde{a}_{f, s}(z+\Delta z, \nu, t)=\tilde{a}_{f, s}(z, \nu, t) \exp \left(-i k_{f, s} \Delta z+\frac{i}{2} k_{f, s} \Delta z \lambda_{f, s}^{2} \nu^{2}\right) .
\end{gathered}
$$

We shall suppose, that the field intensities $\left|a_{f, s}\right|^{2}$ are normalized to $h c / \lambda_{s} \sigma_{g} T_{c a v}$ ( $h$ is the Planck constant; $\lambda_{f}=1.35 \mu \mathrm{m}$ and $\lambda_{s}=1.54 \mu \mathrm{m}$ are the fundamental and Stokes wavelengths, respectively; $\sigma_{g}=0.76 \times 10^{-19} \mathrm{~cm}^{2}$ is the gain cross-section, $T_{\text {cav }}=0.8 \mathrm{~ns}$ ), and the time is normalized to $T_{\text {cav }}$. The gain coefficient is $g$ and its initial value equals to the threshold one: $\left(\ln 1 / T_{0}^{2}+\right.$ $\left.\ln 1 / \rho_{f}+l\right) / 2 L_{g}$. In the last expression, the varied values $T_{0}$ and $\rho_{f}$ correspond, respectively, to the initial transmission of saturable absorber and the reflection of output mirror at the fundamental wavelength; $l=0.05$ is the unsaturable net-loss coefficient. $L_{g}=5 \mathrm{~cm}$ is the active medium length. The stimulated Raman scattering inside an active medium is described by the coefficient $g_{s}$ and its dimensional value amounts to $6 \mathrm{~cm} / \mathrm{GW}$. It is assumed that there exists no anti-Stokes and higher-order Stokes scattering as well as 
that the gain is saturable by only fundamental field. Also, the initial gain distribution is assumed to be spatially homogeneous.

The field representations $\tilde{a}_{f, s}$ within the spatial frequency domain are obtained by the means of the fast Hankel's transformation [13, 14]. $k_{f, s}$ are the wave-numbers corresponding to the fundamental and Stokes fields, respectively.

A similar procedure describes the dynamics inside a saturable absorber:

$$
\begin{aligned}
& \dot{a}_{f}(z, r, t)=-\frac{\Delta z}{2 T_{\text {cav }}} a_{f}(z, r, t)\left[n(r, t)-\sigma_{\text {esa }}(N-n(r, t))\right] \\
& \dot{a}_{s}(z, r, t)=-\frac{\Delta z}{2 T_{c a v}} a_{s}(z, r, t) n(r, t) \\
& \left.\dot{n}(r, t)=-\frac{\sigma_{f} \lambda_{f}}{h c} n(r, t)\left|a_{f}(z, r, t)\right|^{2}-\frac{\sigma_{s} \lambda_{f}}{h c} n(r, t)\left|a_{s}(z, r, t)\right|^{2}+\frac{N-n(r, t)}{T_{r}}\right) \\
& \otimes \tilde{a}_{f, s}(z+\Delta z, \nu, t)=\tilde{a}_{f, s}(z, \nu, t) \exp \left(-i k_{f, s} \Delta z+\frac{i}{2} k_{f, s} \Delta z \lambda_{f, s}^{2} \nu^{2}\right)
\end{aligned}
$$

Here, $n$ is the loss coefficient and its initial value amounts to $N=\left(\ln 1 / T_{0}^{2}\right) / 2 L_{a}$ ( $L_{a}=0.1 \mathrm{~cm}$ is the saturable absorber thickness). The loss is saturable by both fundamental and Stokes fields $\left(\sigma_{f}=95, \sigma_{s}=4\right.$, when the normalization to $\sigma_{g}$ is presumed). Excited-state absorption is taken into account for the fundamental field $\left(\sigma_{\text {esa }}=0.1\right) . T_{r}=26$ is the loss relaxation time (the normalization to $T_{\text {cav }}$ is presumed).

Free propagations between the active medium and the absorber as well as between the active medium and the spherical mirror are considered in the frequency domain:

$$
\tilde{a}_{f, s}(z+L, \nu, t)=\tilde{a}_{f, s}(z, \nu, t) \exp \left(-i k_{f, s} L+\frac{i}{2} k_{f, s} L \lambda_{f, s}^{2} \nu^{2}\right)
$$

where $L=5 \mathrm{~cm}$ is the propagation length.

The reflection from a spherical mirror obeys:

$$
a_{f, s}(z, r, t)=a_{f, s}(z, r, t) \exp \left(i k_{f, s} r^{2} / R_{M}\right)
$$

where $R_{M}$ is the variable radius of the mirror curvature.

The loss on an output mirror as well as the net unsaturable loss are taken into account by means of the following mapping: 
$a$

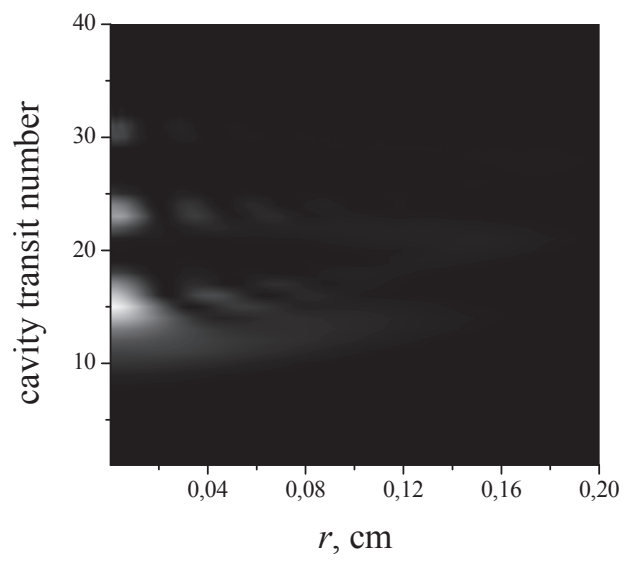

$b$
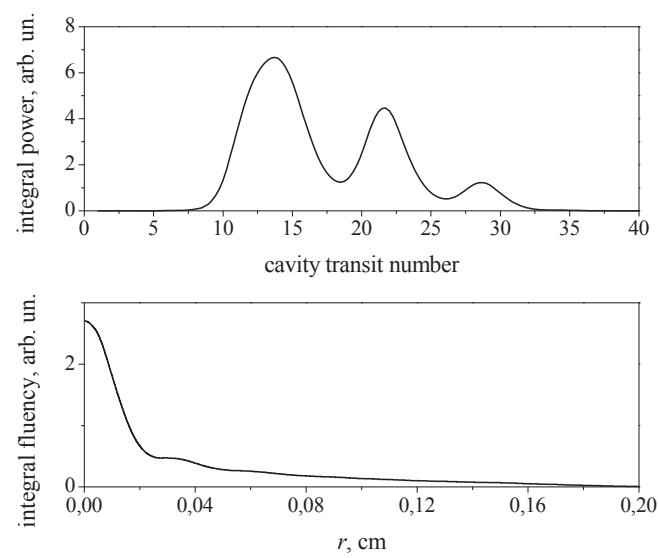

Figure 1: $a$ : Contour plot of the output intensity at the fundamental wavelength from a Q-switched oscillator with $T_{0}=0.3, \rho_{f}=0.7, \rho_{s}=0, R_{M}=300 \mathrm{~cm} . \quad b$ : Output intensity integrated over $r$ (integral power; upper plot) or $t$ (integral fluency; lower plot).

$$
a_{f, s}(z, r, t)=a_{f, s}(z, r, t) \exp \left[-0.5\left(l+\ln \left(1 / \rho_{f, s}\right)\right)\right],
$$

where $\rho_{s}$ is the output mirror reflection at the Stokes wavelength.

In the simulations, the time window is approximated by 100 points and the window of transverse coordinate is approximated by $2000 \div 6000$ points in dependence on the $R$ value, which varies from 0.5 to $1.5 \mathrm{~cm}$ in order to exclude the boundary effects. The solution in the time domain is evaluated using a fourth-order Runge-Kutta method. The initial dimensionless fields have the Gaussian spatial profile with the size, which equals to that of the fundamental $\mathrm{CW}$ mode at $\lambda_{f}$. The initial dimensionless amplitudes $a_{f}$ and $a_{s}$ amount to $10^{-2}$ and $10^{-10}$, respectively.

\section{Spatially-temporal structure of laser field from a Q-switched oscillator}

The simulations based on the model described in the previous Section demonstrate that the spatially-temporal structure of laser field from a Qswitched oscillator depends non-trivially on the oscillator parameters, which effect on the field dynamics at both fundamental and Stokes wavelengths.

Fig. 1, $a$ shows the contour plot of the output intensity at the fundamental wavelength in absence of an effective $\operatorname{SRS}$ in an oscillator $\left(\rho_{s}=0\right)$. The 

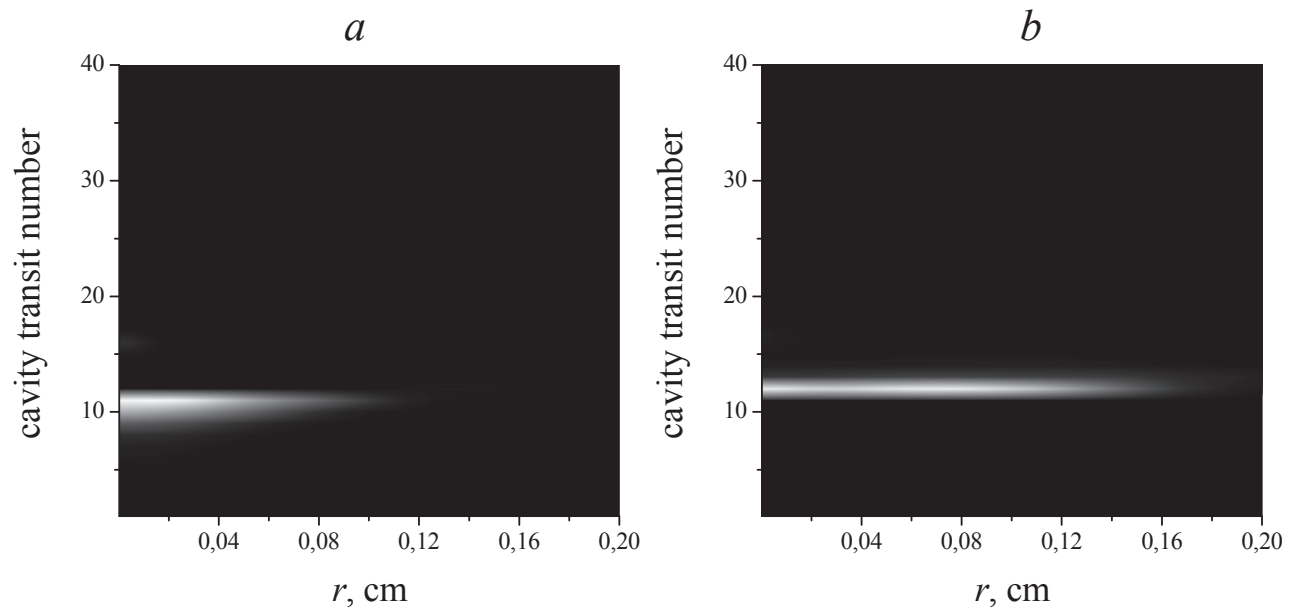

Figure 2: Contour plots of the output intensity at the fundamental $(a)$ and Stokes $(b)$ wavelengths. $T_{0}=0.3, \rho_{f}=0.7, \rho_{s}=0.7, R_{M}=300 \mathrm{~cm}$.

intensities are averaged over the cavity period in Figs. 1, $a$; 2, 4, 5, 7, and 8, Both low initial transmission of a saturable absorber and comparatively small radius of curvature of a spherical mirror initiate the multiple pulse dynamics, which is clearly visible in Fig. 1, $b$ (upper plot) showing the output intensity integrated over the transverse spatial coordinate. The greatest pulse is comparatively long $(\approx 5 \mathrm{~ns})$ and the narrow "mode" (the radius equals to $\approx 130$ $\mu \mathrm{m}$ ) has an elongate wing (lower plot). As will be shown, a non-Gaussian transverse shape is typical for the regime under consideration (see [9]).

When a field at the Stokes wavelength is locked inside an oscillator owing to non-zero $\rho_{s}$, the dynamics changes. For the case presented in Fig. 1, the multipulsing disappears (Figs. 2, $a$ and 3) and the pulse at the fundamental wavelength shortens substantially $(\approx 1.2$ ns, see Fig. 3, upper plot) [10]. The mode at the fundamental wavelength widens and becomes Gaussian (Fig. 3. lower plot; see also [9]). Simultaneously, the short pulse at the Stokes wavelength develops (Figs. 2, $b$ and 3). Such a pulse is located on the tale of the fundamental pulse (Fig. 3, gray curve in upper plot) and has a broad trapezoidal transverse profile (the mode radius reaches $\approx 1.4 \mathrm{~mm}$ in Fig. 3 , gray curve in lower plot).

Thus, the transverse distribution excesses substantially the CW mode size, which equals to $\approx 265 \mu \mathrm{m}$ for the spherical mirror under consideration. Such a spatial extra-broadening can be attributed to the saturable absorber saturation, which results in an excitation of high-order spatial frequencies. 

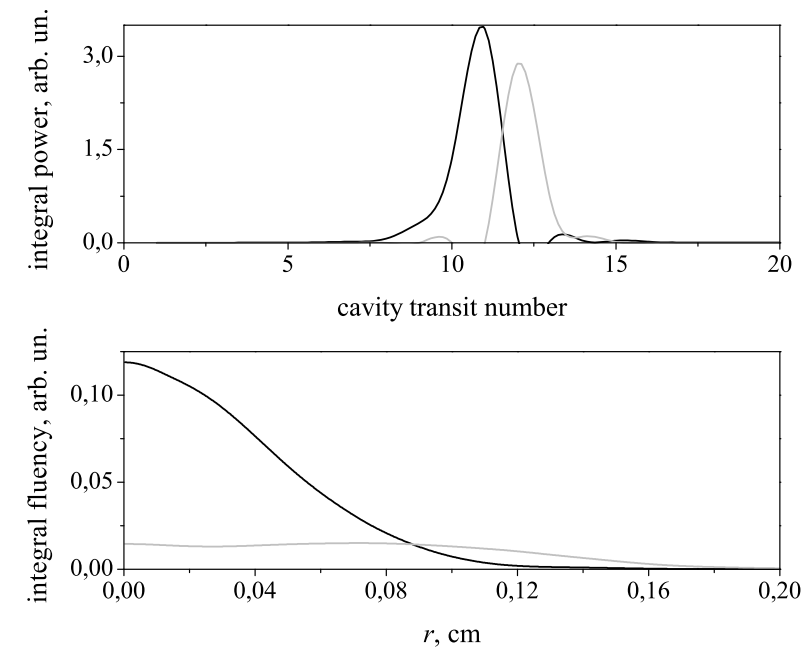

Figure 3: Output integral power (upper plot) and integral fluency (lower plot) at the fundamental (black curves) and Stokes (gray curves) wavelengths. Parameters correspond to Fig. 2

A spatial extra-broadening is possible at the fundamental wavelength, as well. Fig. 4, $a$ demonstrates such an extra-broadening (up to $\approx 0.9 \mathrm{~cm}$ ). The field covers almost a whole active crystal radius. It should be noted, that the output $\mathrm{CW}$ mode radius equals to $\approx 440 \mu \mathrm{m}$ for the spherical mirror under consideration. The Q-switching "mode" has a shape of a dilative ring, which rises and then disappears (after $\approx 12 \mathrm{~ns}$ ) with a gain depletion. In the presence of SRS, such a "mode" is suppressed owing to an inhomogeneous "collapsing" spatially-temporal behavior at both fundamental and Stokes wavelengths (Fig. 4, $b$ and [15]).

An effect of the SRS on the Q-switching dynamics allows controlling the temporal and spatial profiles of a pulse at both fundamental and Stokes wavelengths. An imbalance between the loss and gain saturation at the fundamental wavelength causing the multiple pulsing can be compensated by the nonlinear loss due to SRS. As a result, the well-shaped and substantially shortened [10] pulses with the broad spatial profiles at both wavelengths appear (Figs. 5, 6).

When the oscillator parameters are not optimized, the spatially-temporal structure of the Stokes field can be complicated. For instance, the radial position of the SRS spike can depend on the time (Fig. 7, $a$, where the SRS spikes move off the optical axis with the pulse evolution; this effect 


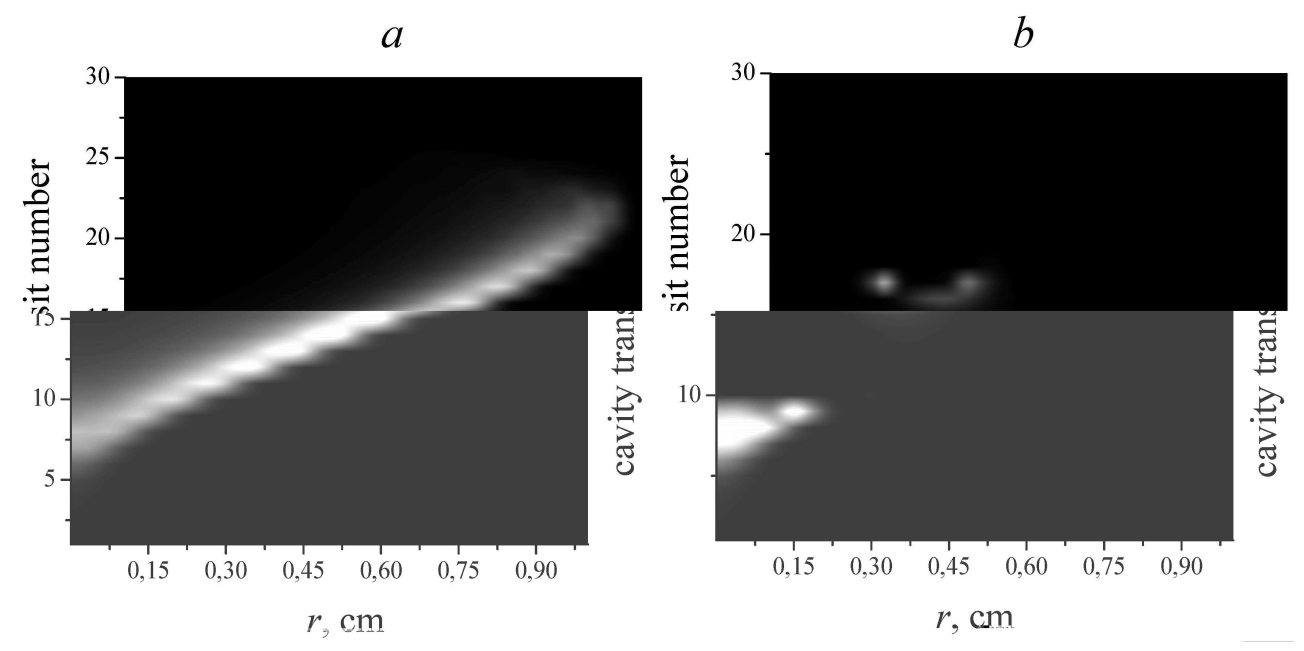

Figure 4: Contour plots of the output intensity at the fundamental wavelength. $T_{0}=0.3$, $\rho_{f}=0.5 ; \rho_{s}=0(a), 0.7(b) ; R_{M}=2000 \mathrm{~cm}$.

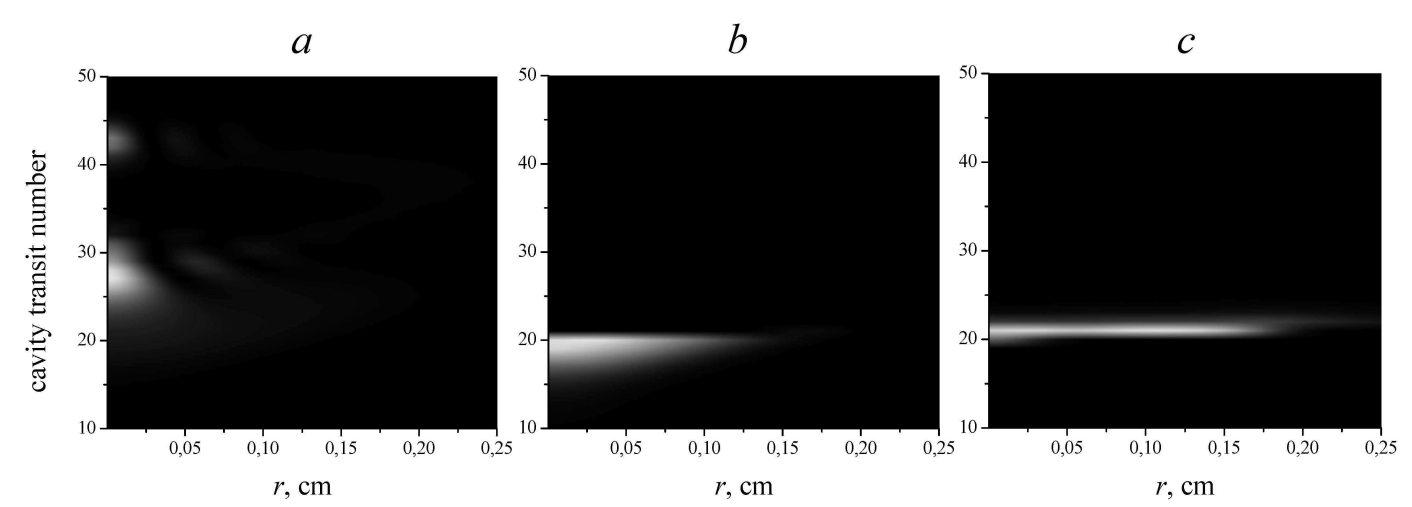

Figure 5: Contour plots of the output intensity at the fundamental $(a, b)$ and Stokes $(c)$ wavelengths. $T_{0}=0.5, \rho_{f}=0.9 ; \rho_{s}=0(a), 0.9(b, c) ; R_{M}=1000 \mathrm{~cm}$. 

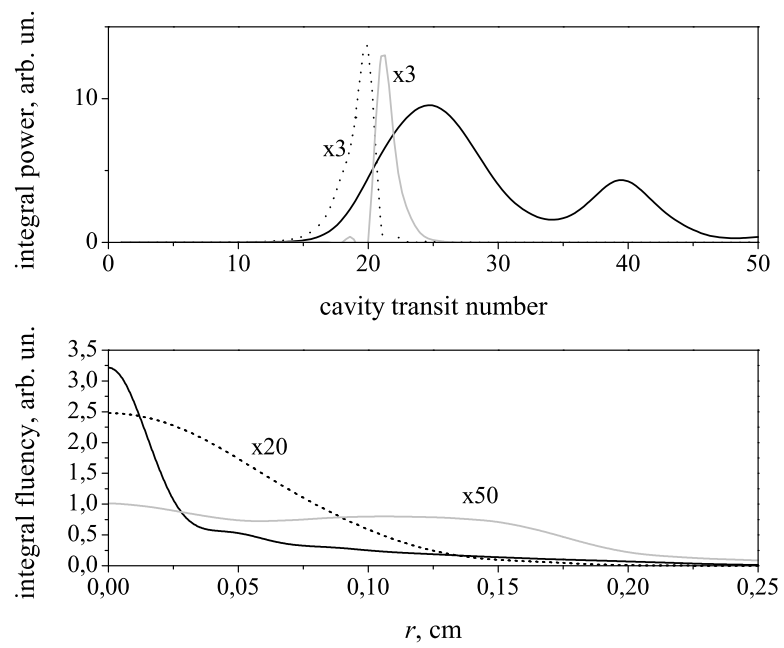

Figure 6: Output integral power (upper plot) and integral fluency (lower plot) at the fundamental (black solid and dotted curves) and Stokes (gray curves) wavelengthes. $\rho_{s}=0$ (black solid curves) and 0.9 (black dotted and gray curves). Another parameters correspond to Fig. 5. Dotted and gray curves are vertically rescaled for convenience.

has been observed experimentally in [16]). A similar situation is possible also at the fundamental wavelength (Fig. 4, a). Even through the SRS is well-synchronized (i.e. the SRS appears synchronously at the different radial positions), the mode profile is inhomogeneous, as a rule (Fig. 7, b).

The oscillator parameters optimization allows regularizing the spatiallytemporal dynamics at both fundamental and Stokes wavelengths. In particular, the spatial profile of the Stokes field becomes homogeneous though non-Gaussian (Fig. 8). The numerical analysis reveals, that there exist some optimal sets of parameters (see Table 1), in particular the optimal curvature of a spherical mirror $(\approx 1000 \mathrm{~cm}$ in the case under consideration), which provide the regular pulses within a broadest range of parameters (viz. $\rho_{f}$, $\rho_{s}$, and $T_{0}$ ). The initial transmission $T_{0}>0.5$ results in a sufficiently strong fundamental field, that causes an efficient SRS. The reflectivity at the Stokes wavelengths has to be sufficiently high (>0.7) to provide an efficient conversion from the fundamental field to the Stokes one.

The resulting pulses at both wavelength are shortened in comparison with a single-wavelength regime and have the smooth and broad transverse spatial profiles. The last property allows using a larger volume of an active crystal. The "mode" shape is close to the Gaussian one for the fundamental field and 

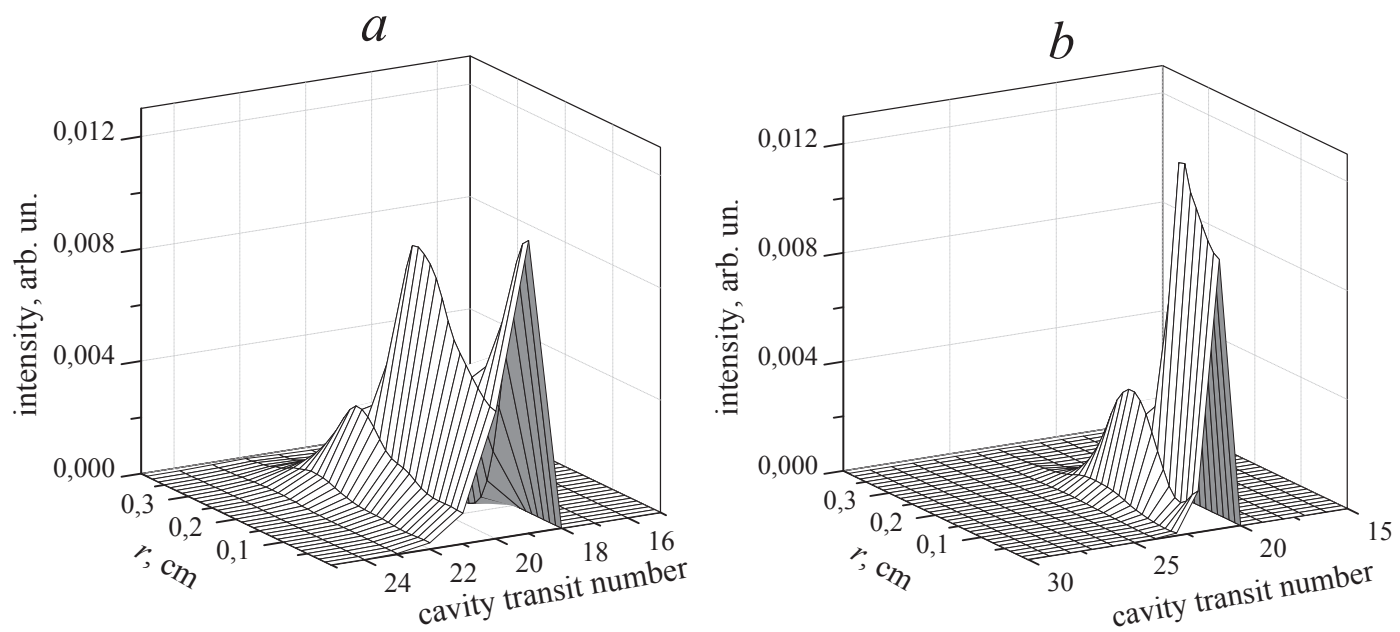

Figure 7: Stokes intensity profiles for $(a): R_{M}=2000 \mathrm{~cm}, \rho_{f}=0.7, \rho_{s}=0.7$, and $(\mathbf{b})$ : $R_{M}=1000 \mathrm{~cm}, \rho_{f}=0.9, \rho_{s}=0.5 . T_{0}=0.5$.

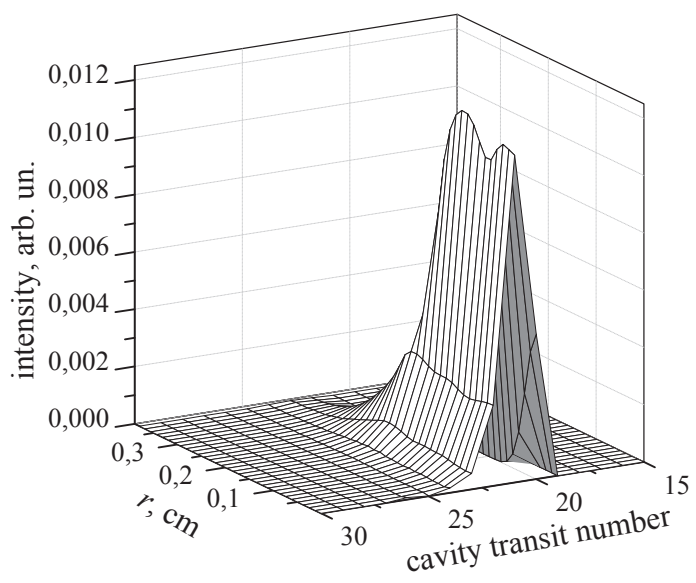

Figure 8: Stokes intensity profile for $R_{M}=1000 \mathrm{~cm}, \rho_{f}=0.9, \rho_{s}=0.9$, and $T_{0}=0.5$. 
Table 1: Oscillator parameters providing the regular spatial-temporal pulse profiles at both fundamental and Stokes wavelengths.

\begin{tabular}{|c|c|c|c|}
\hline$R_{M}, \mathrm{~cm}$ & $T_{0}$ & $\rho_{f}$ & $\rho_{s}$ \\
\hline \hline 300 & $0.3-0.5$ & $0.7-1$ & $0.7-0.9$ \\
\hline 1000 & $0.3-0.5$ & $0.5-1$ & $0.7-0.9$ \\
\hline 2000 & 0.5 & $0.9-1$ & 0.9 \\
\hline
\end{tabular}

to the trapezoidal one for the Stokes field.

\section{Conclusion}

The model of a Q-switched Nd:KGW oscillator with a V:YAG saturable absorber, which takes into account a transverse spatial field distribution at both fundamental and Stokes wavelengths, has been developed. The numerical analysis has demonstrated that an imbalance between the loss and gain saturation causes not only a multiple pulsing but an aberration of an oscillator "mode". When the initial transmission of a saturable absorber is sufficiently low, the spatial extra-broadening of a field develops so that a "mode" covers almost whole volume of an active crystal.

Manipulations with the beam size on a saturable absorber (by means of the change of a spherical mirror curvature), the initial transmission of an absorber, and the output mirror reflectivity at both fundamental and Stokes wavelengths allow controlling the spatially-temporal profiles of the output pulses. To obtain a single pulsing at two-wavelengths with a broad and homogeneous spatial distribution, the initial transmission of the saturable absorber has to be about of 0.5 , the reflectivity of the output mirror at the fundamental wavelength has to approach 1 (it can range within $0.5-1$ for the optimal curvature of the spherical mirror, which is of $\approx 1 \mathrm{~m}$ in the case under consideration), and the reflectivity at the Stokes wavelength has to be about of 0.9 . The SRS causes a temporal squeezing of the pulses at both wavelengths, whereas the spatial profiles are greatly stretched in comparison with the $\mathrm{CW}$-mode size. When the oscillator parameters are optimized, the transverse spatial profiles are near-Gaussian at the fundamental wavelength and trapezoidal at the Stokes wavelength.

The obtained results are of interest for development of the passively Qswitched Raman-active oscillators producing the pulses with durations of 
about of few nanoseconds and possessing the smooth transverse spatial profiles covering a considerable part of an active crystal.

\section{References}

[1] G.J.Spuhler, R.Paschotta, R.Fluck, B.Braun, M.Moser, G.Zhang, E.Gini, U.Keller, J. Opt. Soc. Am. B 16 (1999) 376.

[2] A.M.Malyarevich, V.G.Savitski, P.V.Prokoshin, N.N.Posnov, K.V.Yumashev, E.Raaben, A.A.Zhilin, J. Opt. Soc. Am. B 19 (2002) 28.

[3] I.J.Miller, A.J.Alcock, J.F.Bernard, in: OSA Proc. Advanced SolidState Lasers, vol. 13, 1992, p.322.

[4] J.Findeisen, H.J.Eichler, P.Peuser, Optics Commun. 181 (2000) 129.

[5] A.A.Lagatsky, A.Abdolvand, N.V.Kuleshov, Opt. Lett. 25 (2000) 616.

[6] A.S.Grabtchikov, A.N.Kuzmin, V.A.Lisinetskii, V.A.Orlovich, A.A.Demidovich, K.V.Yumashev, N.V.Kuleshov, H.J.Eichler, M.B.Danailov, Optical Meterials 16 (2001) 349.

[7] A.S.Grabtchikov, A.N.Kuzmin, V.A.Lisinetskii, V.A.Orlovich, and G.I.Ryabtsev, Appl. Phys. Lett. 75 (1999) 3742.

[8] A.S.Grabtchikov, A.N.Kuzmin, V.A.Lisinetskii, V.A.Orlovich, A.A.Demidovich, M.B.Danailov, H.J.Eichler, A.Bednarkiewicz, W.Strek, and A.N. Titov, Appl. Phys. B 75 (2002) 795.

[9] W.Chen, Y.Inagawa, T.Omatsu, M.Tateda, N.Takeuchi, Y.Usuki, Optics Commun. 194 (2001) 401.

[10] V.L.Kalashnikov, Optics Commun. 218 (2003) 147.

[11] Y.V.Loiko, A.A.Demidovich, V.V.Burakevich, A.P.Voitovich, J. Opt. Soc. Am. B 22 (2005) 2450.

[12] Sh.Ding, X.Zhang, Q.Wang, J.Zhang, Sh.Wang, Y.Liu, X.Zhang, J. Physics D 40 (2007) 2736.

[13] A.E.Siegman, Lasers (University Science Books, 1986). 
[14] M.Guizar-Sicairos, J.C.Gutierrez-Vega, J. Opt. Soc. Am. A 21 (2004) 53.

[15] V.L.Kalashnikov, http://info.tuwien.ac.at/kalashnikov/presentation7/index.htm

[16] A.P.Shkadarevich, V.I.Dashkevich, V.A.Orlovich, in: N.S.Kazak, P.A.Apanasevich, V.V.Kabanov, S.N.Kurilkina, V.Ju.Plavskij, S.G.Rusov (Eds.), Proc. VII International Conference on Laser Physics and Optical Technologies, v.3, Minsk, Belarus, 2008, p. 477 (in Russian). 